\title{
Polycyclic aromatic hydrocarbons as markers of environmental and hydrological processes (identification problems and research experience)
}

\author{
Aleksandr Khaustov $^{1,}$, , Margarita Redina ${ }^{1}$, and Inna Nemirovskaya ${ }^{2}$ \\ ${ }^{1}$ RUDN-University, Ecological Faculty, 117198 Miklukho-Maklaya st. 6, Moscow, Russia \\ ${ }^{2}$ Shirshov Institute of Oceanology of RAS, 117997, Nahimovskiy pr. 36, Moscow, Russia
}

\begin{abstract}
The efficiency of the study of polycyclic aromatic hydrocarbons (PAHs) as markers of many environmental and hydrological processes is disclosed. On the example of the interaction between sea and river water the flows of substances and problems of their detection in media, as well as the laws of fractionation during phase transitions at geochemical barriers (GCB) are presented. The authors have collected an extensive database for almost all types of natural environments. Own research was conducted at specialized water testing sites (springs, a mud volcano, brine of a lake, the Northern Dvina River, water of Black and White Seas, water bodies of the Moscow Region and Albena region (Bulgaria), etc. This allowed substantiating the optimal markers and creating effective methods for identifying a complex of hydrological processes.
\end{abstract}

\section{Introduction}

Analysis of PAHs environmental pathways allows us to clearly identify processes in the media and identify sources of pollution: technogenic (industrial, transport), natural (biological synthesis and destruction of materials). Despite the extremely low concentrations of PAHs in the media ( $\mu \mathrm{g}$ and $\mathrm{ng} / \mathrm{l}$ ), the scale of their migration is impressive. Identification of PAHs is faced with a complex of problems of sampling, storage, sample preparation and imperfection of analytical methods [1]. This makes their detection expensive and still exotic for Russia. However, these studies are extremely important and provide high efficiency in the understanding of hydrological and hydrochemical processes. The most complex are the definitions of PAHs for the aquatic environment, which is the basis for the migration of solutions in the elements of ecosystems and forms natural and technogenic flows. Experience with PAHs suggests their effectiveness in identifying the following hydrological processes.

1. Assessments of river run-off in "pure" and technogenic pressured areas; evaluations of genetic types of river and lake alimentation during various periods of water regime.

\footnotetext{
* Corresponding author: khaustov-ap@rudn.ru
} 
2. Identification of the genesis and scales of water pollution (pyrogenic, petrogenic, biogenic, caused by specific industries); search for the pollution source (incl. natural discharges of hydrocarbons).

3. Calculations of the wastewater dilution using the numerical models of turbulent diffusion; thermodynamics of manifestation and transformation forms of organic pollutants in water.

4. Monitoring and postmonitoring of the accidental oil spills at water bodies.

5. The movement of suspended matter in sections of rivers, lakes, seas, sedimentation rates of organomineral sediments, diffusion processes in bottom sediments, the identification of the geochemical boundaries in the system "water - bottom sediments".

6. Assessment of stratification in the interaction of river and sea waters, clarification of the size of hydrochemical zones of marginal filters (according to A.P. Lisitsin [2]), identification of substances transport processes.

Let us dwell on the last task - identification of the processes of transport of substances in river zones (Northern Dvina - White Sea), physicochemical interaction and biological absorption, since in this example we can consider most of the problems of poor knowledge of environmental processes in river mouths.

\section{Research methods and objects}

Objects of study are the components of the aquatic system "river - sea" of the Northern Dvina river. The data were obtained from 3 stations, in the river zone (station $3 \mathrm{a}$ ), in the gravitational and physical-chemical zones of the marginal filter (station 11) and in the sea zone (station 2a) [3]. The dynamics of mass transfer in the studied zones were analyzed using the data on PAHs distribution. Their concentration coefficients $\mathrm{CC}$ were analyzed for cases of the transition through GCB (ratio of $\Sigma \mathrm{PAH}$ in the "receiving" medium to the $\Sigma \mathrm{PAH}$ in the releasing one). For all studied media, the most probable sources (pyrogenic, petrogenic) of the pollution were identified using traditional indicator ratios $[1,4,5]$.

\section{Results and discussion}

\subsection{Aquatic system of the marginal filter: river zone}

Conditions of the migration of PAHs within the river in are presented by the following contatcs: "snow - ice", "water - ice", "water-bottom"; "ice (bottom edge) - ice (upper edge)", "water (depth 0.0 - $5.0 \mathrm{~m}$ ) - water (depth 5.0-14.0 m)" (Fig. 1). The maximal $\Sigma \mathrm{PAH}$ is detected in the bottom sediments $(47800 \mathrm{ng} / \mathrm{kg})$, the minimal one - in the river water (till $0.015 \mathrm{ng} / \mathrm{dm}^{3}$ ). The PAHs flow intencity according to the CC formed the sequence: "water - bottom"> "ice (bottom edge) - ice (upper edge)" > "water $(5.0 \mathrm{~m})$ - ice (bottom edge)" > "water $(14.0 \mathrm{~m})$ - water $(5.0 \mathrm{~m})$ ". Under the conditions of turbulence of the river water, such model is quite justified. Low $\Sigma$ PAH in the stream are caused by its isolation from external pollutants from snow and ice, and secondly, by preferential supply of groundwater, protected from aerogenic pollution.

The migration of PAHs through the GCB is selective. Pl, Flu, Py dominated in the snow, but the kinetic PAHs (Flu, An) moved to the upper edge of the ice, and Naph, which is similar in behavior to them, as well as thermodynamic (Phen, Py). At the "water - ice" interface, the active kinetic migrants showed more contrast in the following sequence (on intensity): $\mathrm{An}>\mathrm{Naph}>\mathrm{BaP}>\mathrm{Chr}>\mathrm{Phen}>\mathrm{Flu}$. With an increase in ice cover in river conditions, PAH migration increases, and from lower concentrations to higher, almost 5 times. Migration in the direction of the negative concentration gradient may be due to the pulsating nature of the interaction at the "water - ice" barrier. 


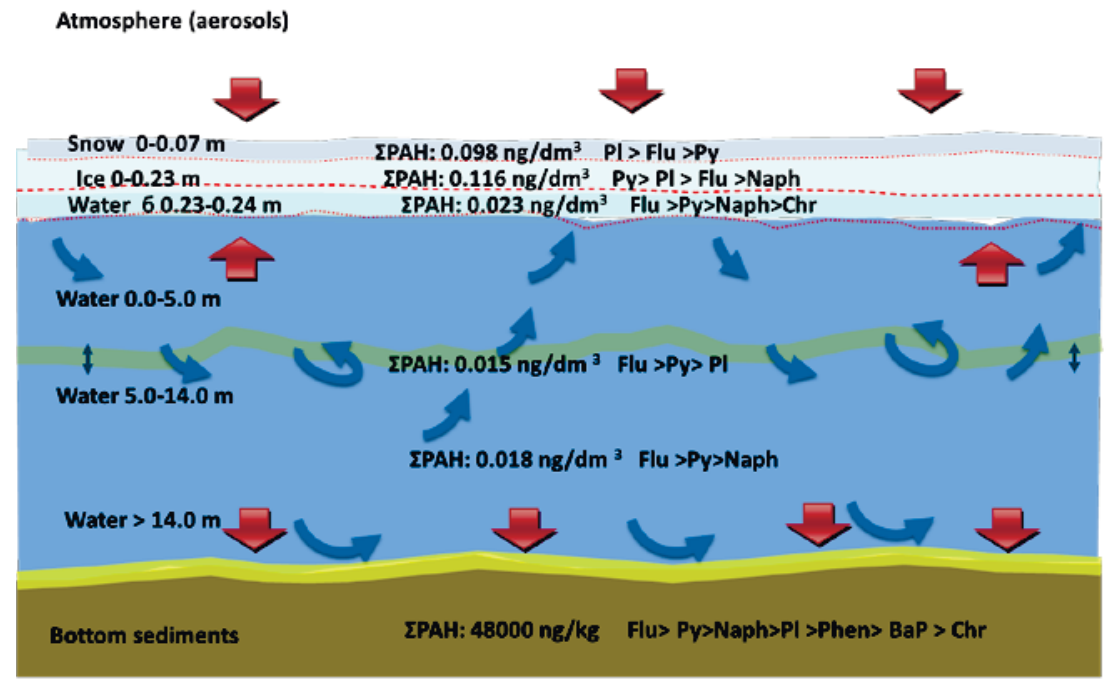

Fig. 1. Scheme of PAH interactions at geochemical barriers in the river zone, station $3 \mathrm{a}$.

An important conclusion can be made: the ice cover in the period of ice freezing on the rivers, due to the pulsation of flows and interaction with the already formed ice, forms its own association of migrants differing from that prevailing in the aquatic environment.

The homogeneity of the PAHs composition within the water column allows justifying the absence of the water stratification in the river conditions. Bottom sediments, which act as a "deposition medium," are radically different from other phase media judging by the averaged $\Sigma$ PAHs $(47.8 \mu \mathrm{g} / \mathrm{kg})$. According to existing norms (i.e. Veileder for klassifisering av miljøgifter i vann og sediment TA-2229/2007), such concentrations correspond to a very dangerous pollution level; marker ratios indicate its mixed nature (both oil and pyrogenic).

In the series of PAHs migration on the GCB "water - bottom sediment", Phen, Py, and Naph are clearly leading; this indicates the identity of the composition of bottom waters and the active water exchange between these components of the aquatic system. The last two PAHs are due to the technogenic input of ship emissions and fuel combustion products. In general, for this barrier we can talk about the typomorphism and paragenesis of PAHs.

\subsection{Aquatic system of the marginal filter: gravitational physical-chemical zone of interaction of sea and river waters}

The general role of this zone is the quantitative and qualitative conversion of matter flows. The interactions of fresh and brackish waters at the beginning of the zone dominate (at salinity 1-5\%o). Here, the concentration of suspended matter is higher than in the final mixing areas of river and sea waters. The intensive development of coagulation and flocculation processes contributes to the active transition of organic substances from solution to suspension. At a salinity of about $2 \%$, a "silt plug" arises in estuaries (areas with abnormally high suspension contents and plankton development) a "biological plug".

In the area of the station 11 (one of the mouth channels of the Northern Dvina river), all the $\mathrm{CC}$ for the lower and upper ice edges were below 1. This indicates indirectly a fundamentally different nature of its formation. The ice is formed by river waters under conditions of backwater with a sharp slowdown in the river flow rates and with an increase in the interaction time of fresh portions of water. Judging by the PAHs composition, ice is intensive polluted with predominantly pyrogenic substances. According to the CC values, the following series of migration intensities with the "lower - upper ice edges" system is 
formed: BaP $(1.43)>$ Naph $(0.84)>$ An $(0.73)>$ Phen $(0.67)>$ Flu $(0.57)>$ Py $(0.51)>\mathrm{Pl}$ $(0.50)>\mathrm{Chr}(0.02)$. The relatively more water-soluble Naph, Py, Pl, and Flu are extracted from the dissolved forms of migration in suspension with further sinking to the bottom and become less accessible for participation in cryogenesis (Fig. 2).

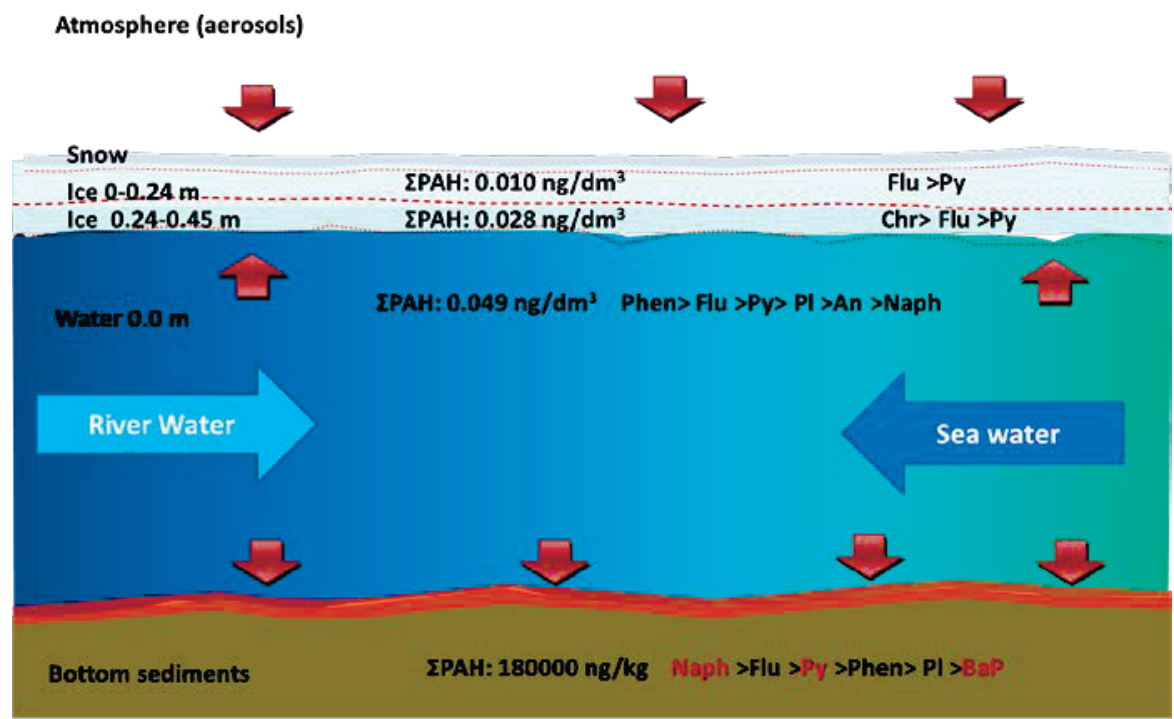

Fig. 2. Scheme of PAH interactions at geochemical barriers, art. 11, the gravitational and physicalchemical zones of the marginal filter.

Summing up, in this zone of the marginal filter, the following patterns can be noted.

- Within the GCB "snow-ice", flows of predominantly technogenic PAHs are active due to aerosols with a predominance of Flu, Py and Naph.

- In the lower part of the ice, an increase in $\Sigma$ PAH occurs due to the duration of contact and stable ice formation, which leads to the accumulation of $\mathrm{Chr}$.

- Between the lower and upper edges, only one active migrant was identified - BaP, which indicates the potential ability to migrate within the barriers.

- The flow of PAHs from water to ice forms specific associations of PAHs with the representation of almost all of them, but not all of them are "absorbed" by ice, some of them are absorbed by biota at the border of the barrier.

- Water between the ice and the bottom forms its association of PAHs with the predominance of Flu, Phen, Py, and An, which indicates the formation of non-stratified strata with the presence of natural and technogenic PAHs.

- The PAHs flow to bottom sediments is significantly different from their combination in dissolved form, which indicates the ability to fractionate them in suspensions. The dominant role is played by $\mathrm{Naph}, \mathrm{BaP}$, Flu, and $\mathrm{Chr}$, mainly by technogenic PAHs.

- It is in the gravitational zone of the marginal filter that the maximum content of suspended matter with PAHs adsorbed on them was recorded.

\subsection{Aquatic system of the marginal filter: biological zone}

The specifics of the hydrological and hydrochemical situation at station $2 \mathrm{a}$ is due to its location in the "throat" (estuary) of the White Sea. Ice cover exists here seasonally, with a maximum in February - March. In the vicinity of the station, the maximum amount of suspensions was recorded in the snow; their concentration significantly changed depending on the time of sampling $[1,5]$. PAH concentrations in snow were almost $50 \mathrm{ng} / \mathrm{dm}^{3}$ higher 
than in ice, although the chemical composition of the dominants is almost the same. The $\mathrm{CC}$ values showed that only for An the transition from cryosols to the ice phase is possible, although hydrocarbon markers in the snow (according to Fly and Py ratios) indicate a fresh pyrogenic pollution (Fig. 3).

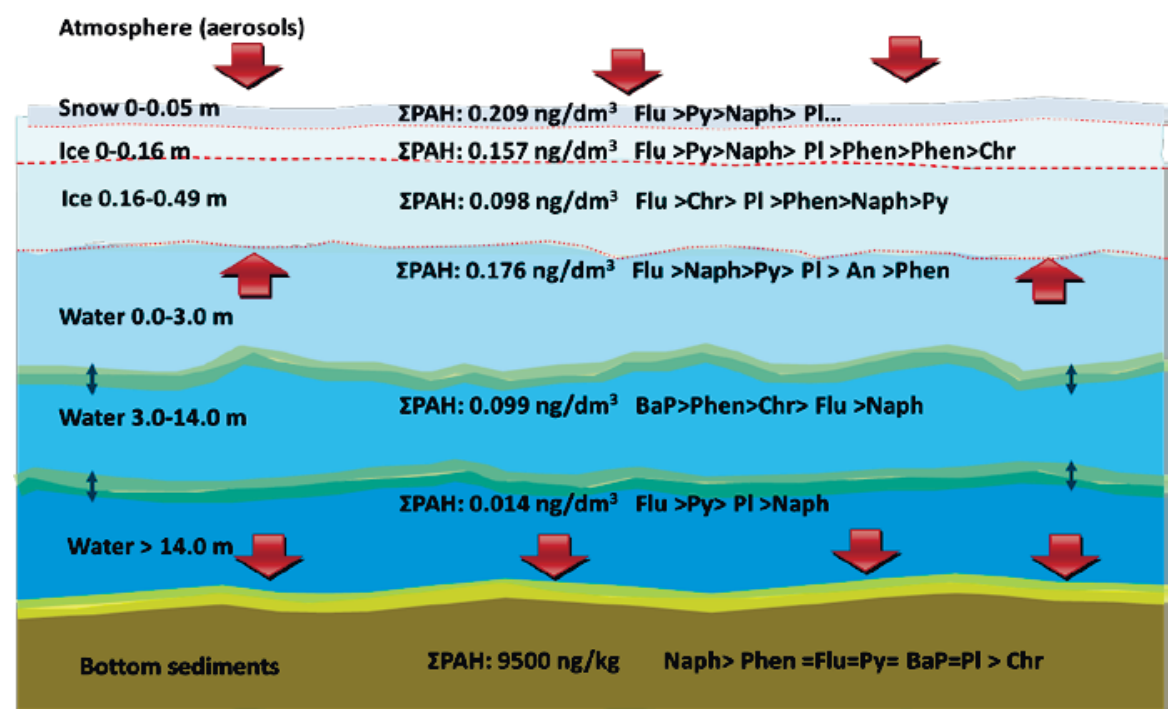

Fig. 3. Scheme of PAH interactions at geochemical barriers in the marine part of the bay, station 2a, marginal filter biological zone.

In the system "snow - sea ice" at the station 2a, the largest amount of PAHs was recorded in snow (horizon 0-5 cm): $\Sigma$ PAH amounted to $209 \mathrm{ng} / \mathrm{dm}^{3}$ with the prevalence of Flu, Py, and Naph. Upon transition to the ice formation (the top edge of the ice to $16 \mathrm{~cm}$ ), the concentrations of almost all compounds sharply decrease, with the exception of Phen, An, BaP, Bghi. $\Sigma$ PAH decreased to $157 \mathrm{ng} / \mathrm{dm}^{3}$. Judging by $\mathrm{CC}$, the only PAH migrant in the "snow - ice" system is An (1.32). The increase in the concentration of PAHs in the snow cover with distance from the source can be explained by their long-range transport in the atmosphere in suspension with northerly winds.

For ice, ice suspensions is distinguished as a component of the subsystem (intermediate stage of the "water - ice" transition). They contain a special composition of PAHs and organomineral substances that have passed from water or the atmosphere to ice during its formation. The composition of the suspension of the upper part of the ice is significantly different even at stations close to each other. The ratios of PAH concentrations in the upper part of the ice suspension and the lower ice part were: Naph (4.0) $>\mathrm{Chr}(2.0)>$ Phen $=\mathrm{An}$ (1.5). The composition of the PAHs in the upper part of the ice suspension cannot be explained only by snow pollution. However, Naph accumulates in the upper part of the ice suspension, as well as $\mathrm{Chr}$ and Phen coming from the lower part of the ice suspension. Unambiguously, only the maximum amount of Flu $(\mathrm{CC}=6.0)$ comes from snow.

An analysis of vertical changes in CC values in the system "water surface - lower edge of ice" clearly indicates that the largest structural changes of PAHs in the composition of ice occur when water interacts with its lower edge. Chr can be attributed to active migrants at the "water - ice" interfaces within the marine part and in the backwater zone. In the river zone, the list of PAHs - applicants for the transition to the ice phase is expanding due to the most active migrant An $(\mathrm{CC}=65.0)$; with a significant margin, Naph followed by $\mathrm{Chr}$, Py and Phen. Judging by the amount of PAHs and CC, water layers from $14 \mathrm{~m}$ and below practically do not come into contact with the ice cover, despite possible vertical inversions. 
In river estuaries, a clear stratification of water is recorded, due to several layers with different densities and temperatures. For the upper less dense layers $(0-3 \mathrm{~m})$, the maximum $\Sigma$ PAH content was recorded: $0.176 \mathrm{ng} / \mathrm{dm}^{3}$. This indicates their formation due to river (more polluted) waters. A clear stratification of the waters is well reflected by a change in the composition of PAHs together with a decrease in $\Sigma \mathrm{PAH}$ to $0.014 \mathrm{ng} / \mathrm{dm}^{3}$ at the bottom at a depth of more than $14 \mathrm{~m}$. Under conditions of technogenesis, the flows of PAHs to the bottom change significantly in composition; Naph and Flu begin to prevail.

\section{Conclusion}

1. The spatial and temporal distribution of PAHs within the aquatic system of the "river-sea" marginal filter indicates the objective existence and the most important functional purpose of this structure. A significant role is played by GCB of various genesis in the redistribution of material and energy flows, which allows us to consider them as an "active medium" capable of spatiotemporal self-organization. Barriers can be considered as active centers of evolution of aquatic geochemical systems.

2. The use of data on organic substances (even at the level of phenomenological models) fundamentally changes the understanding of the relationship between the components in the hydrological and geochemical system. Identification of homophase and heterophase media and assessment of the interaction and behavior of energy-material flows is a necessary methodological technique.

3. Calculations show: stable PAH associations do not exist. All of them are more or less susceptible to transformation, where the maximum is reached on the barriers-sections [6].

4. Extremely active (with a change in concentrations of tens of thousands of times) the absorption and conversion of PAHs occur with the participation of living matter. The inclusion of this substance in the scheme of interaction of aquatic systems elements radically changes the idea of the functional significance of biota in the structure of flows.

5. The overall "rating" of PAH concentration activity at the GCB borders within the marginal filter of Northern Dvina river can be represented as a series: "seston - water"> "separation suspension - water" > "bottom sediments (contaminated) - water"> "bottom sediments (relatively clean) - water"> "ice top edge - ice bottom edge (sea conditions)", etc.

The material has been prepared in the framework of the 5-100 project, financially supported by the Ministry of Education and Science of the Russian Federation (the Agreement № 02.A03.21.0008).

Presented models are built on the base of data obtained in the RFBR projects 17-05-00356, 18-0580049 and modeling approaches developed in the RFBR project 07-05-07046.

\section{References}

1. A. P. Khaustov, M. M. Redina., Geochemistry International, 55(1), 98-107 (2017).

2. A. P. Lisitsyn, Okeanologiya, 34(5), 735-747 (1994).

3. I. A. Nemirovskaya, Oil in the ocean. Pollution and natural flows (Moscow, Nauchny mir, 2013) (in Rus.).

4. A .P. Khaustov, RUDN J. of Ecol. and Life Safety, 25(3), 396-413 (in Rus.).

5. I. A. Nemirovskaya, A. P. Khaustov, M. M. Redina, Izvestiya Rossiiskoi Akademii Nauk. Seriya Geograficheskaya, 6, 49-56 (2018) (in Rus.).

6. A.Yu. Opekunov, Environmental sedimentology (Saint-Petersburg Univ. Publ., Saint-Petersburg, 2012) (in Rus.). 